http://dx.doi.org/10.32929/2446-8355.2019v28n4p493-504

\title{
CAPACIDADES FISIOLÓGICAS E PRODUÇÃO DE MATÉRIA SECA DE ACESSOS DE BATATA-DOCE BATATA-DOCE
}

\author{
Evander Alves Ferreira ${ }^{1}$, Gustavo Antônio Mendes Pereira ${ }^{2 *}$, Deivide Patrik Alves ${ }^{3}$, Luciane \\ Renata Agazzi Agazzi ${ }^{4}$, Daniel Valadão Silva ${ }^{5}$, Valter Carvalho de Andrade Júnior ${ }^{6}$ \\ ${ }^{1}$ Docente, Matologia, Universidade Federal de Minas Gerais, Montes Claros-MG. \\ 2 Pós-doutorando, Matologia, Universidade Federal de Viçosa, Rio Paranaíba-MG. *E-mail do autor \\ correspondente: gustavogamp@hotmail.com \\ ${ }^{3}$ Graduando, Matologia, Universidade Federal de Viçosa, Rio Paranaíba-MG. \\ ${ }^{4}$ Mestre, Matologia, Universidade Federal de Viçosa, Viçosa-MG. \\ ${ }^{5}$ Docente, Matologia, Universidade Federal Rural do Semi-Árido, Mossoró-RN. \\ ${ }^{6}$ Docente, Olericultura, Universidade Federal de Lavras, Lavras-MG.
}

Recebido: 01/07/2019; Aceito: 25/11/2019

RESUMO: Existem vários fatores que afetam a fotossíntese, como o déficit físico, o estresse térmico, a concentração de gás interno e externo e a intensidade da luz, no entanto, também pode variar com o material genético ou cultivar avaliado. $\mathrm{O}$ objetivo deste estudo foi avaliar as características fisiológicas de setenta e sete genótipos de batata-doce e agrupá-los de acordo com sua produção de matéria fisiológica e seca. Para este fim, foi estabelecido um delineamento de blocos ao acaso (DBC). As características fisiológicas foram coletadas 60 dias após o transplante das mudas de batata-doce nas folhas mais expandidas contadas a partir do meristema apical do ramo mais longo. Foi utilizado um analisador de gás nãoinfravermelho (IRGA). Com o uso deste dispositivo foi avaliado: a concentração de $\mathrm{CO}_{2}(\mathrm{Ci})$ e a taxa fotossintética (A), a condutância estomática dos vapores de água (Gs), a taxa de transpiração (E), a eficiência do uso da água (EUA) e a proporção Ci / carbono atmosférico (Ci / Ca). Aos 150 dias após o transplante, determinou-se a massa de matéria seca total das plantas (MST). Os genótipos de batata-doce avaliados apresentaram comportamento diferente em relação às diferentes características fisiológicas estudadas. Valores mais elevados de A, Gs, Ci, Ci / Ca e E foram observados em UFVJM-57, mas UFVJM-06 mostrou os valores mais baixos em relação a essas características.

Palavras-chave: Ipomoea batatas. Fotossíntese. Condutância estomática. Uso eficiente da água.

\section{PHYSIOLOGICAL ASPECTS OF SWEET POTATO ACCESSIONS}

ABSTRACT: There are several factors that affect the substance or chemical such as a physical deficit, thermal stress, internal and external gas concentration and light intensity, however, it can also vary with the evaluated genetic or cultivar material. The objective of this study was to evaluate the physiological characteristics of seventy-seven sweet potato genotypes (Ipomoea batatas) and to group them according to their physiological and dry matter production. For this purpose, a randomized block disassembly (DBC) test was 
established. As physiological comments were made 60 days after the transplantation of the sweet potato seedlings in the most expanded leaves counted from the apical meristem of the longer branch. A non-infrared gas analyzer (IRGA) was used. The use of this device was evaluated: the concentration of sub-static $\mathrm{CO} 2(\mathrm{Ci})$ and the photosynthetic rate $(\mathrm{A})$, stomatal conductance of water vapors (Gs), transpiration rate (E), water use efficiency (USA) and the ratio $\mathrm{Ci} /$ atmospheric carbon $(\mathrm{Ci} / \mathrm{Ca})$. At 150 days after transplanting, the total dry matter mass of the plants (MST) was determined. The evaluated sweet potato genotypes presented different behavior in relation to the different physiological characteristics studied. Higher values of $\mathrm{A}, \mathrm{Gs}, \mathrm{Ci}, \mathrm{Ci} / \mathrm{Ca}$ and $\mathrm{E}$ were observed in UFVJM-57, but UFVJM-06 showed the lowest values in relation to these characteristics.

Key words: Ipomoea batatas. Photosynthesis. Stomatal conductance. Water use efficiency.

\section{INTRODUÇÃO}

O cultivo de batata-doce (Ipomoea batatas (L.) Lam.) tem sido apontado como alternativa promissora para incrementar a produção de bioenergia (etanol) (SANTANA et al., 2013) e de alimento para pecuária, em especial para bovinos (ANDRADE JÚNIOR et al., 2012). De acordo com Rukundo et al. (2013) e Andrade Júnior et al. (2014) destacam que ramas e raízes de batata-doce podem ser integradas aos materiais vegetais convencionais e proporcionar nutrição pecuária com qualidade e oferta, além de ser utilizada na alimentação humana.

Porém, o cultivo de batata-doce no Brasil e sua utilização, principalmente para os fins supracitados, são mal aproveitados. A produtividade nacional é da ordem de $12 \mathrm{t} \mathrm{ha}^{-1} \mathrm{e}$ em função da rusticidade dos materiais, as plantas são cultivadas em áreas marginais, muitas vezes sem adubação complementar ou técnicas de plantio (ANDRADE JÚNIOR et al., 2009; VARGAS et al., 2017). Adicionalmente, existe grande número de acessos cultivados, que apresenta grande variabilidade genética e fenotípica (ANDRADE JÚNIOR et al., 2014), fatores que dificultam as recomendações para cultivo. A batata-doce apresenta custo de produção relativamente baixo e retorno elevado sendo matéria prima para obtenção de álcool, amido, pães, doces, farinhas, flocos e féculas (SANTOS et al., 2009).

Vários fatores limitam a produção da cultura da batata-doce, os principais são baixa fertilidade do solo, ataque de insetos, doenças e condições climáticas desfavoráveis, influenciando o crescimento vegetal e a produção. Destaca-se também os aspectos fisiológicos que são limitantes ao aumento de produtividade. A fotossíntese é afetada direta ou indiretamente por diversos fatores como, déficit hídrico, estresse térmico, concentração interna e externa de gases e composição e intensidade da luz (TAIZ et al., 2017), no entanto, ela pode variar também de acordo com o material genético ou cultivar avaliado (FERREIRA et al. 2011). A taxa fotossintética está diretamente relacionada à radiação fotossinteticamente ativa, aos fatores de disponibilidade hídrica, e às trocas gasosas. A fotoinibição, definida como a redução na eficiência fotossintética dependente da luz, está diretamente associada à disponibilidade de $\mathrm{CO}_{2}$ e de radiação fotossinteticamente ativa (CONCENÇO et al., 2007). 
Em condições naturais onde as plantas estão sujeitas a diversas variações e diversos outros fatores ambientais, os mecanismos relacionados às características fotossintéticas das culturas são complexos, destacando que essas características podem variar de acordo com os genótipos ou biótipo estudado (FERREIRA et al., 2011).

Dessa forma, objetivou-se com o presente estudo avaliar as características fisiológicas de setenta e sete genótipos de batata-doce e agrupando-as de acordo com suas capacidades fisiológicas e produção de matéria seca.

\section{MATERIAL E MÉTODOS}

O experimento foi instalado em condições de campo no Setor de Olericultura, Campus JK da Universidade Federal dos Vales do Jequitinhonha e Mucuri (UFVJM), em DiamantinaMG, (18²'01'’S; 4334'20'’O; $1.400 \mathrm{~m}$ de altitude) entre os anos de 2012 e 2013.

O regime climático da região é tipicamente tropical, $\mathrm{Cwb}$ na classificação de Köppen, a precipitação média anual varia de 1.250 a $1.350 \mathrm{~mm}$ e a temperatura média anual do ar situase na faixa de $18^{\circ} \mathrm{C}$ a $20^{\circ} \mathrm{C}$, com umidade relativa do ar quase sempre elevada, revelando médias anuais de $70,6 \%$.

Foi utilizado uma irrigação automática, programada para funcionamento por período de 30 minutos às 8 horas da manhã e 16:00 horas da tarde, com vistorias diárias para evitar excesso ou falta de água, e a homogeneidade em todos as parcelas. As unidades experimentais foram compostas por 6 linhas espaçadas de 0,5 metros com comprimento de 6 metros e foram mantidas sob temperatura e iluminação naturais durante a condução do experimento, os dados climáticos no período da execução estão apresentados na Figura 1.

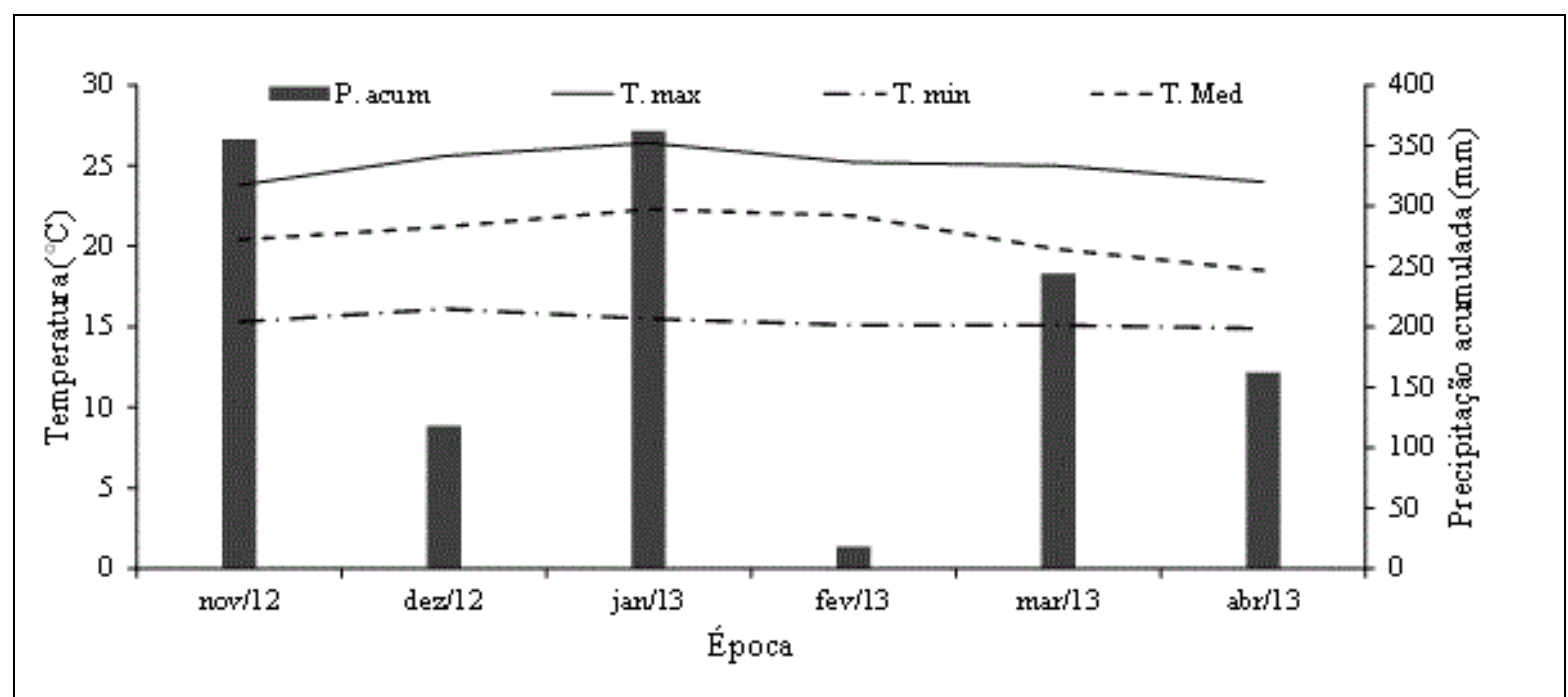

Figura 1. Temperaturas máxima (T. max), média (T. med) e mínima (T. min) e precipitação acumulada (P. acum) no local e período de realização do experimento. Diamantina, MG, 2012/2013./ Maximum temperatures (T.max), mean (T.med) and minimum (T.min) and accumulated precipitation (P.acum) at the and period of the experiment. Diamantina, MG, 2012/2013.

Fonte: INMET. INMET. 
Os tratamentos constaram de genótipos de batata-doce cultivados no Setor de Olericultura do Departamento de Agronomia da Universidade Federal dos Vales do Jequitinhonha e Mucuri. O experimento foi montado no delineamento em blocos casualizados (DBC), sendo alocados setenta e dois tratamentos representado pelas genótipos: Tomba carro 1, UVJM-01, UVJM-28, Cariru vermelha, UVJM-41, UVJM-21, UVJM-25, UVJM-23, Cambraia, Brazlandia rosada, UVJM-18, UVJM-57, UVJM-40, UVJM-31, Princesa Licuri, UVJM-39, UVJM-04, UVJM-14, UVJM-36, UVJM-30, Palmas, UVJM-06, UVJM-20, Brazlandia branca, UVJM-16, UVJM-19, Tomba carro 2, UVJM-47, UVJM-49, UVJM-10, UVJM-56, UVJM-03, UVJM-35, UVJM-46, UVJM-33, Marmel, UVJM-05, UVJM-13, UVJM-17, UVJM-42, UVJM-37, UVJM-09, UVJM-24, UVJM-38, UVJM-44, Arruba, Espanhola, UVJM-48, UVJM-29, UVJM-26, UVJM-62, UVJM-12, UVJM-07, UVJM-43, UVJM-08, UVJM-02, UVJM-32, UVJM-45, UVJM-27, UVJM-50, Batata mandioca, UVJM51, UVJM-59, UVJM-52, UVJM-53, UVJM-58 UVJM-34, UVJM-60, UVJM-55, UVJM-22 e UVJM-54, com quarto repetições.

As ramas para produção de mudas foram obtidas do banco de germoplasma da UFVJM, sendo cortadas em estacas com quatro a oito nós, plantadas em bandejas de isopor com substrato comercial para hortaliças (Bioplant Plus Germinação - Composição: turfa de sphagnum, fibra de coco, casca de arroz, casca de pinus e vermiculita) e mantidas em estufa, sob sombrite de $50 \%$ de insolação. O transplantio das mudas para o campo foi realizado manualmente. A adubação de cobertura foi realizada 30 dias após o plantio aplicando-se 30 $\mathrm{kg} \mathrm{ha}^{-1}$ de $\mathrm{N}$ (150 kg ha ${ }^{-1}$ de sulfato de amônio). A irrigação por aspersão foi feita desde o plantio, nos momentos onde se achou necessário o fornecimento. $\mathrm{O}$ experimento foi montado em um Neossolo Quartizarênico órtico típico, antes da implantação do experimento é: $\mathrm{pH}$ (água) - 5,8; P e K (mg dm $\left.{ }^{-3}\right)$ - 1,1 e 6,3 respetivamente; $\mathrm{SB}, \mathrm{Ca}, \mathrm{Mg}, \mathrm{Al}$ e $\mathrm{H}+\mathrm{Al}$ (cmolc dm ${ }^{-}$ 3) - 1,1;0,6;0,5;0,2 e 2,4 respetivamente; V (\%) - 32; MO, areia, argila e silte $\left(\mathrm{dag}_{\mathrm{kg}}^{-1}\right)-$ 0,86, 6 e 8 respectivamente. O solo foi corrigido e adubado de acordo com análise de solo, sendo fornecidos $1,2 \mathrm{t} \mathrm{ha}^{-1}$ de calcário dolomítico.

As avaliações dos aspectos fisiológicos foram realizadas aos 30, 60 e 120 dias após o transplantio das mudas de batata-doce nas folhas adultas da batata-doce, correspondente a folha mais expandida contada do meristema apical da rama mais longa. Foi utilizado um analisador de gases no infravermelho (IRGA), marca ADC, modelo LCA 4 (Analytical Development Co. Ltd, Hoddesdon, UK), em casa de vegetação aberta, permitindo livre circulação do ar. Foram avaliadas a concentração de $\mathrm{CO}_{2}$ subestomática $\left(\mathrm{Ci}-\mu\right.$ mol mol$\left.{ }^{-1}\right)$ e a taxa fotossintética $\left(\mathrm{A}-\mu \mathrm{mol} \mathrm{m} \mathrm{m}^{-2} \mathrm{~s}^{1}\right.$ ), a condutância estomática de vapores de água (Gs mol m $\left.\mathrm{m}^{-1} \mathrm{~s}^{-1}\right)$, a taxa de transpiração $\left(\mathrm{E}-\mathrm{mol} \mathrm{H}_{2} \mathrm{O} \mathrm{m}^{-2} \mathrm{~s}^{-1}\right)$, a eficiência no uso da água (EUA $\mu \mathrm{mol} \mathrm{CO}_{2} \mathrm{mmol}^{-1} \mathrm{H}_{2} \mathrm{O}=$ fotossíntese/ transpiração) e a relação carbono interno/carbono atmosférico $(\mathrm{Ci} / \mathrm{Ca})$.

Cada bloco foi avaliado em um dia, entre 8 e 10 horas da manhã, em dia de céu limpo e com iluminação natural, de forma a manter as condições ambientais homogêneas durante a avaliação de cada bloco. Em cada unidade experimental, foram avaliadas a planta central e uma planta periférica escolhida ao acaso. 
Para determinação na massa da matéria seca total (MST), as plantas foram coletadas aos 150 dias após o plantio, colocadas em sacos de papel, transportadas para o Laboratório de Olericultura do Departamento de Agronomia da UFVJM e colocadas em estufa de circulação forçada de ar a $60^{\circ} \mathrm{C}$ até massa constante.

Os dados foram submetidos à análise de variância pelo teste $\mathrm{F}$ em nível de $5 \%$ de probabilidade, sendo as medias agrupadas pelo Critério de Agrupamento de Scott-Knott a 5\% de probabilidade utilizando o software SISVAR 5.6 ${ }^{\circledR}$.

\section{RESULTADOS E DISCUSSÃO}

Os setenta e dois genótipos de batata-doce apresentaram diferenças marcantes para todas as variáveis fisiológicas avaliadas, sendo os mesmos, agrupados de acordo com a variável em questão pelo Critério de Agrupamento de Scott-Knott.

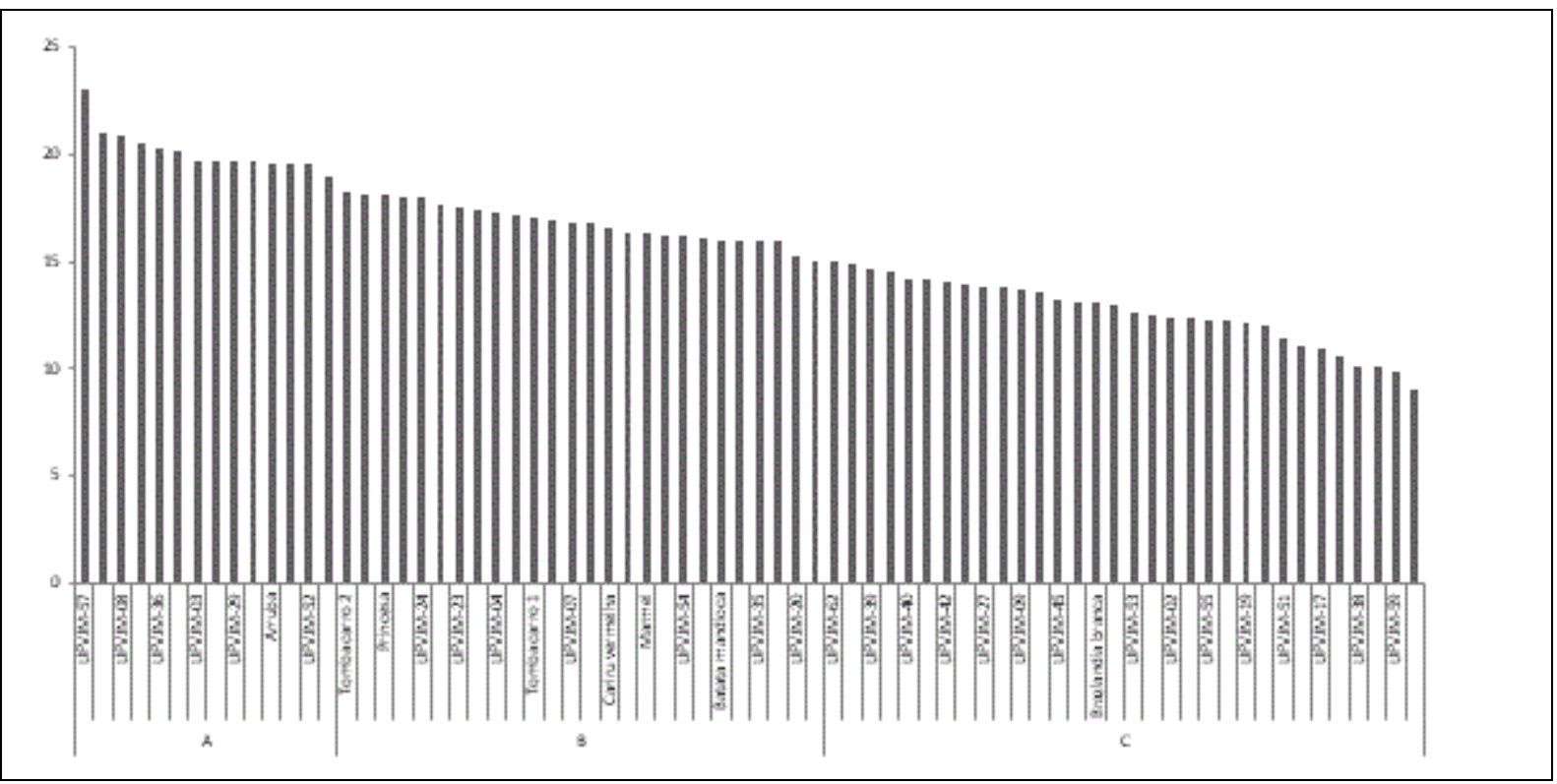

Figura 2. Taxa fotossintética $\left(\mathrm{A}-\mu \mathrm{mol} \mathrm{m} \mathrm{m}^{-2} \mathrm{~s}^{-1}\right)$ de setenta e dois genótipos de batata-doce cultivadas no Alto Vale do Jequitinhonha, Diamantina-MG. Médias seguidas pela mesma letra maiúscula em cada barra, não diferem entre si pelo Critério de agrupamento de ScottKnott a 5\% de probabilidade. Diamantina, MG. / Photosynthetic rate $\left(A-\mu \mathrm{mol} \mathrm{m} \mathrm{m}^{-2} \mathrm{~s}^{-1}\right)$ of seventy and seven sweet potato genotypes cultivated in the Alto Vale do Jequitinhonha, Diamantina-MG. Mediums followed by the same capital letter in each bar, do not differ from each other by the Scott-Knott grouping criterion to $5 \%$ of probability. Diamantina, MG.

Fonte: Autoria própria. Own authorship.

Ao avaliar a taxa fotossintética (A) genótipos de batata-doce verificou-se que o genótipo UFVJM-57 apresentou o mais alto valor de A, sendo agrupada juntamente com outros 13 genótipos com elevados valores de $\mathrm{A}$, nesse grupo a A variou de 18 a $23 \mu \mathrm{mol} \mathrm{m}^{-2} \mathrm{~s}^{-1}$, para as cultivares UFVJM-32 e UFVJM-57, respectivamente. Considerando que segundo o mesmo Critério foram formados três agrupamentos, sendo o segundo grupo formado por 26 genótipos, nesse grupo os valores de A variaram de 15 (UFVJM-58) a $18 \mu \mathrm{mol} \mathrm{m}^{-2} \mathrm{~s}^{-1}$ (Tomba carro 2), já último grupo com 32 genótipos esses valores variaram de aproximadamente 9,00 (UFVJM-25) a 15,00 $\mu \mathrm{mol} \mathrm{m} \mathrm{m}^{-2} \mathrm{~s}^{-1}$ (UFVJM-62) (Figura 2). Ferreira et al. (2011) trabalhando 
com 10 cultivares de cana-de-açúcar verificaram diferenças marcantes na taxa fotossintética e em outras variáveis fisiológicas relacionadas a essa espécie, tais como, condutância estomática, taxa transpiratória e carbono interno.

Com relação à condutância estomática (Gs) de genótipos de batata-doce, constatou-se a formação de quatro grupos pelo Critério de Scott-Knott. O genótipo de batata-doce UFVJM57, também apresentou o maior valor referente à variável fisiológica Gs, aproximadamente 1,45 $\mathrm{mol} \mathrm{m}^{-1} \mathrm{~s}^{-1}$, ou seja, $40 \%$ superior ao valor observado para o segundo genótipo com maior abertura estomática, o UFVJM-01 com $0,85 \mathrm{~mol} \mathrm{~m}^{-1} \mathrm{~s}^{-1}$. Assim o primeiro grupo apresentou 15 genótipos, o segundo 25, o terceiro 21 e o último grupo 11 genótipos, sendo que, esses valores variaram de 0,08 (UFVJM-06) a 1,45 $\mathrm{mol} \mathrm{m}^{-1} \mathrm{~s}^{-1}$ (UFVJM-57) (Figura 3).

A condutância foliar é composta em pequena parte pela condutância cuticular da epiderme, e quando os estômatos estão abertos, pela condutância estomática que é controlada majoritária da abertura estomática. Assim, a condutância é proporcional ao número e tamanho dos estômatos e diâmetro da abertura do estômato, características que dependem de outros fatores endógenos e ambientais (CONCENÇO et al., 2008).

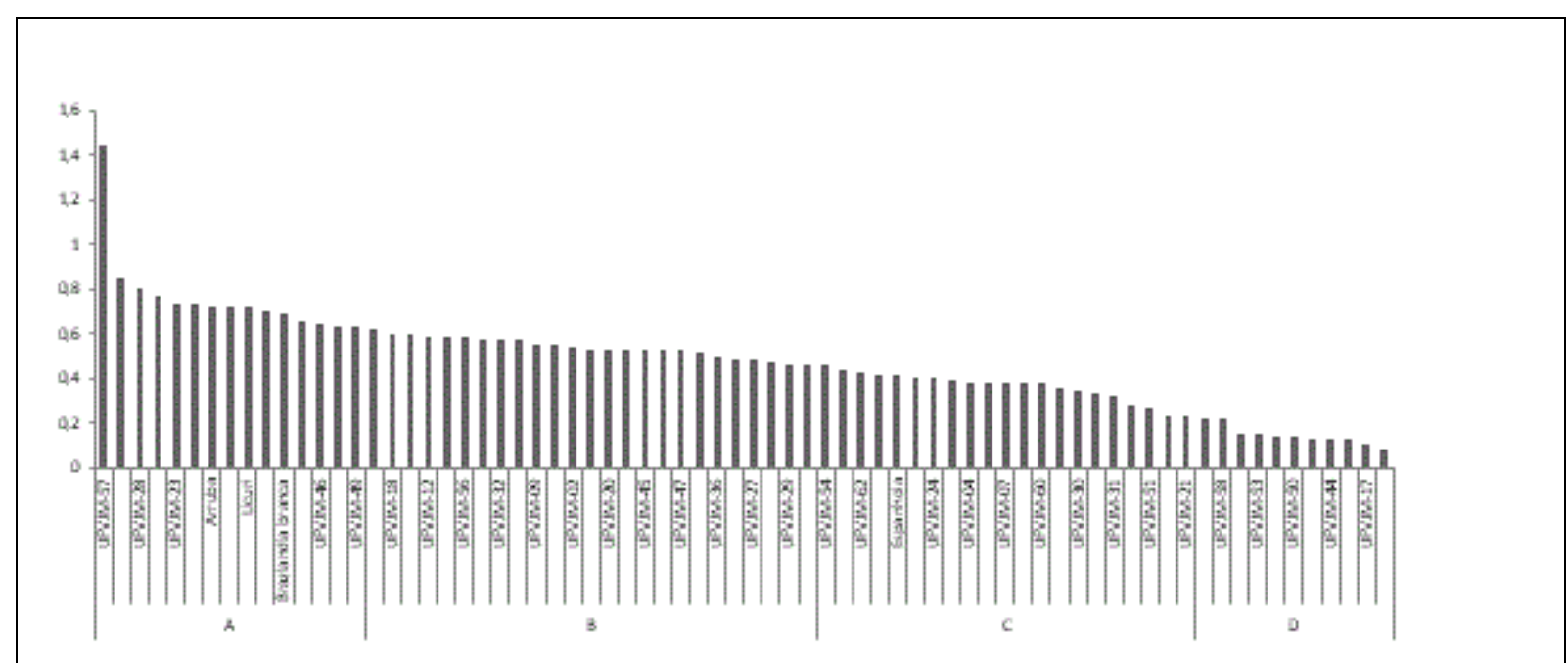

Figura 4. Carbono interno $\left(\mathrm{Ci}-\mu \mathrm{mol} \mathrm{mol}^{-1}\right)$ de setenta e sete genótipos de batata-doce cultivadas no Alto Vale do Jequitinhonha, Diamantina-MG. Médias seguidas pela mesma letra maiúscula em cada barra, não diferem entre si pelo Critério de agrupamento de ScottKnott a 5\% de probabilidade. Internal carbon $\left(\mathrm{Ci}-\mu \mathrm{mol} \mathrm{mol} \mathrm{l}^{-1}\right)$ of seventy-seven sweet potato genotypes grown in the Alto Vale do Jequitinhonha, Diamantina-MG. Means followed by the same capital letter in each bar do not differ by Scott-Knott's Grouping Criterion at 5\% probability.

Fonte: Autoria própria. Own authorship.

Os materiais genéticos UFVJM-21 e UFVJM-57 apresentaram os maiores valores referentes à relação carbono interno/carbono atmosférico $(\mathrm{Ci} / \mathrm{Ca})$, correspondentes a 0,91 , no entanto esses valores não mostraram diferença estatística em relação a 45 genótipos agrupados segundo o Critério de Agrupamento de Scott-Knott. Assim foi formado segundo esse critério de agrupamento mais dois grupos com 17 e 8 genótipos, respectivamente, destacando-se que o menor valor observado para essa variável foi 0,5 , para o material genético UFVJM-06 (Figura 5). 


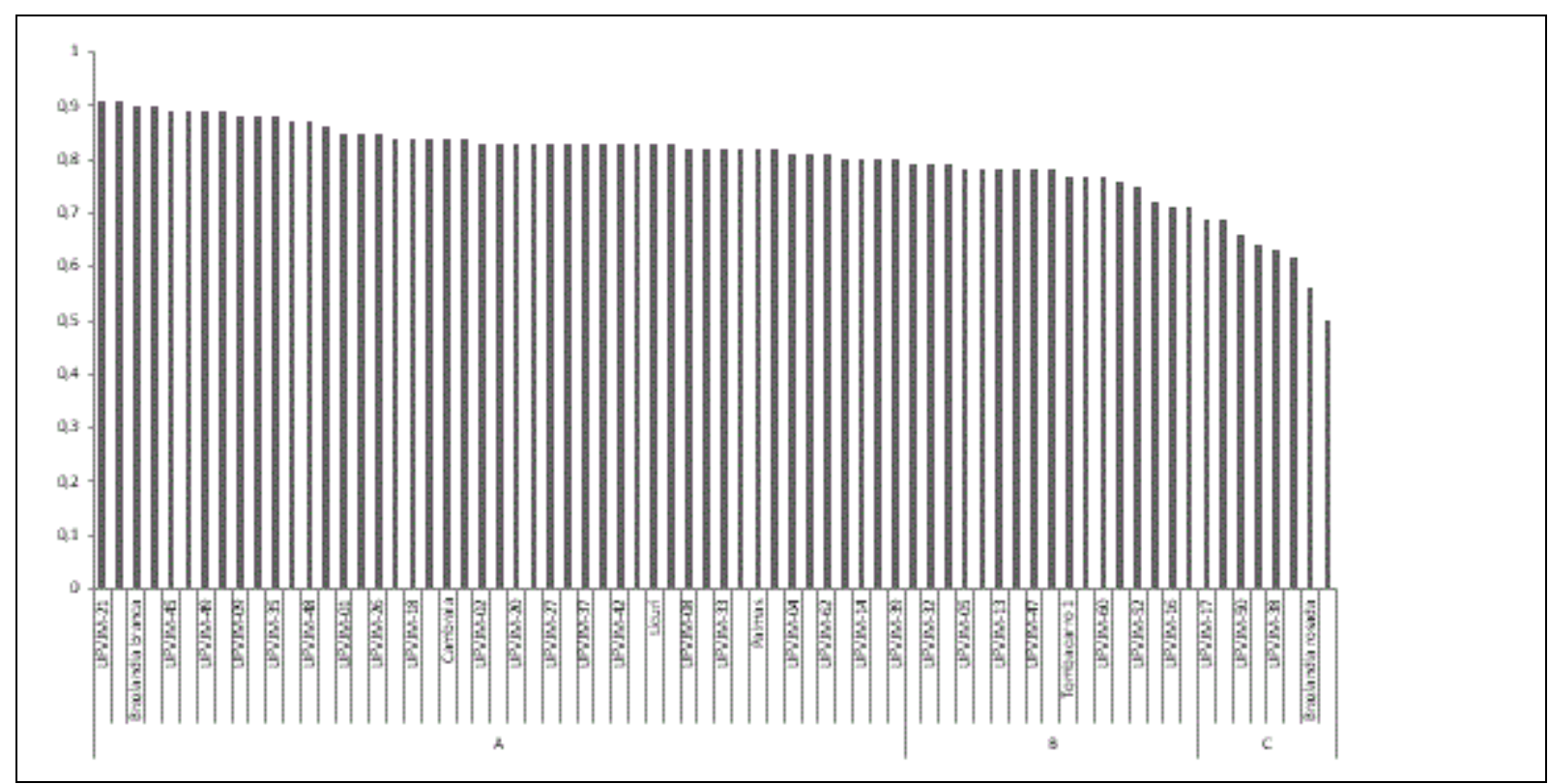

Figura 5. Relação carbono interno/carbono atmosférico $(\mathrm{Ci} / \mathrm{Ca})$ de setenta e dois genótipos de batata-doce cultivadas no Alto Vale do Jequitinhonha, Diamantina-MG. Médias seguidas pela mesma letra maiúscula em cada barra, não diferem entre si pelo Critério de agrupamento de Scott-Knott a 5\% de probabilidade. Inner carbon / atmospheric carbon ratio ( $\mathrm{Ci} / \mathrm{Ca}$ ) of seventy-seven sweet potato genotypes grown in the Alto Vale do Jequitinhonha, DiamantinaMG. Means followed by the same capital letter in each bar do not differ by Scott-Knott's Grouping Criterion at 5\% probability.

Fonte: Autoria própria. Own authorship.

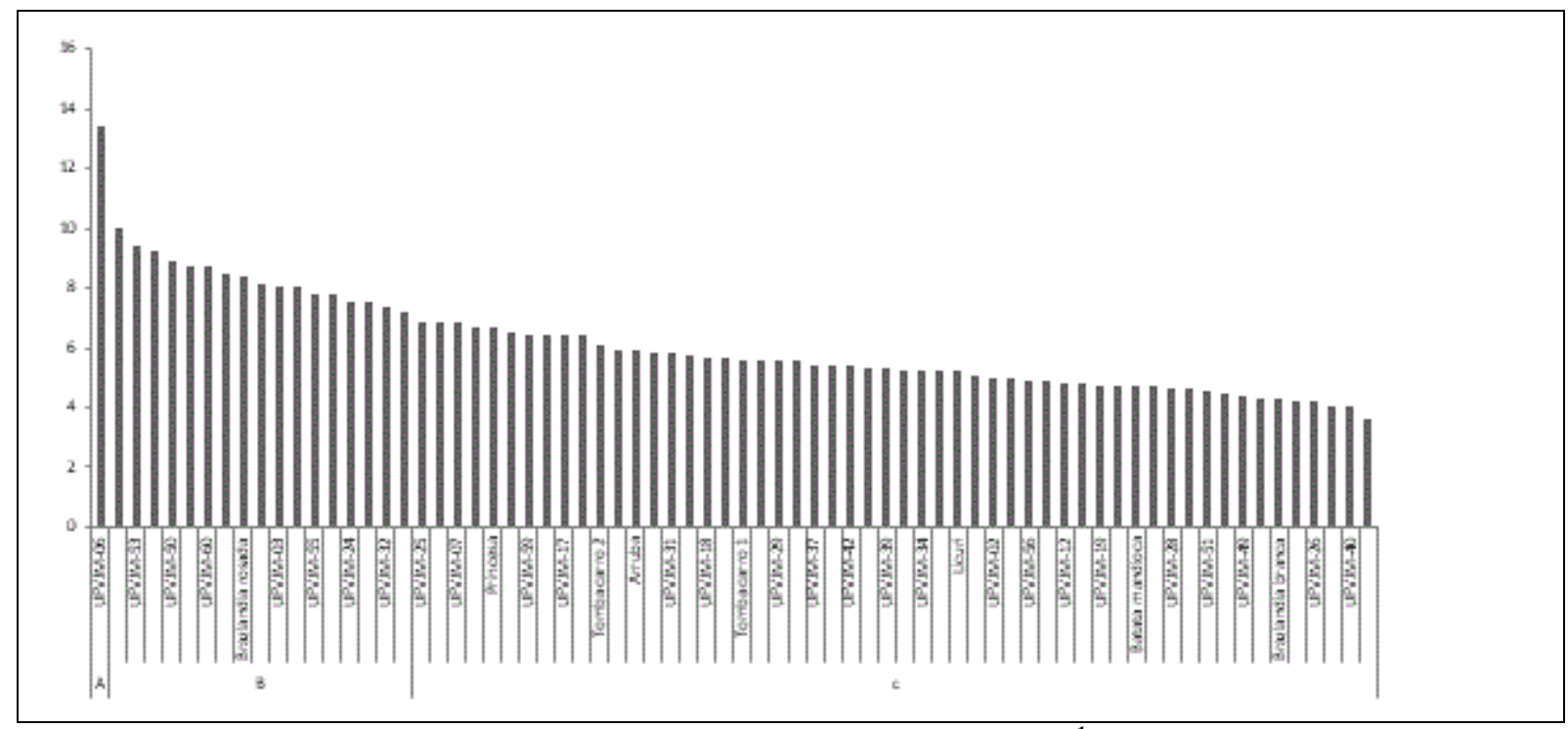

Figura 6. Eficiência no uso da água (EUA - mol $\mathrm{CO}_{2} \mathrm{~mol} \mathrm{H}_{2} \mathrm{O}^{-1}$ ) de setenta e dois genótipos de batata-doce cultivadas no Alto Vale do Jequitinhonha, Diamantina-MG. Médias seguidas pela mesma letra maiúscula em cada barra, não diferem entre si pelo Critério de agrupamento de Scott-Knott a 5\% de probabilidade. Efficiency in water use (USA - mol $\mathrm{CO}_{2} \mathrm{~mol} \mathrm{H}_{2} \mathrm{O}^{-1}$ ) of seventy-seven sweet potato genotypes grown in the Alto Vale do Jequitinhonha, DiamantinaMG. Means followed by the same capital letter in each bar do not differ by Scott-Knott's Grouping Criterion at 5\% probability.

Fonte: Autoria própria. Own authorship. 
Com relação à eficiência no uso da água (EUA) observou-se a formação de três grupos de genótipos de acordo com o critério de agrupamento utilizado, sendo que, o primeiro grupo apresentou um único genótipo com elevado valor da EUA, UFVJM-06 $\left(13,4 \mathrm{~mol} \mathrm{H}_{2} \mathrm{O} \mathrm{m}^{2} \mathrm{~s}^{-}\right.$ $\left.{ }^{1}\right)$, sendo esse valor cerca de $30 \%$ superior ao encontrado para a cultivar UFVJM-52 (10,02 mol $\mathrm{H}_{2} \mathrm{O} \mathrm{m}^{2} \mathrm{~s}^{-1}$ ) agrupada no segundo agrupamento juntamente com outros 16 materiais genéticos. O último grupo mostra 59 genótipos com valores de EUA variando de 3,64 a 6,84

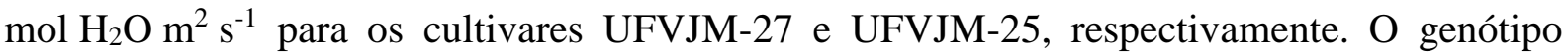
UFVJM-57 que mostrou maiores valores de A, apresentou a EUA de 5,21 $\mathrm{mol} \mathrm{H}_{2} \mathrm{O} \mathrm{m}^{2} \mathrm{~s}^{-1}$, ou seja, um valor intermediário se comparado aos demais materiais genéticos (Figura 6).

A razão entre a taxa fotossintética e a taxa de transpiração resulta na eficiência do uso da água (EUA), que é mais um parâmetro fisiológico que expressa quantitativamente o comportamento momentâneo das trocas gasosas na folha e que varia entre e dentro das espécies vegetais (CONCENÇO et al., 2007; PINZÓN-TORRES e SCHIAVINATO, 2008).

O uso eficiente da água pode ser conceituado como a quantidade de água transpirada pela planta para a produção de determinada quantidade de matéria seca (SILVA et al., 2012). Sendo assim, plantas com maiores valores de EUA tende a ter maior capacidade de produzir matéria seca por grama de água transpirada. O EUA possui correlação direta com o tempo de abertura estomática, pois, ao abrir os estômatos para assimilar $\mathrm{CO}_{2}$ para realizar a fotossíntese, a água é perdida por transpiração, com intensidade variável, sendo dependente do gradiente de potencial entre a atmosfera e a superfície foliar, seguindo uma corrente de potenciais hídricos (CONCENÇO et al., 2007).

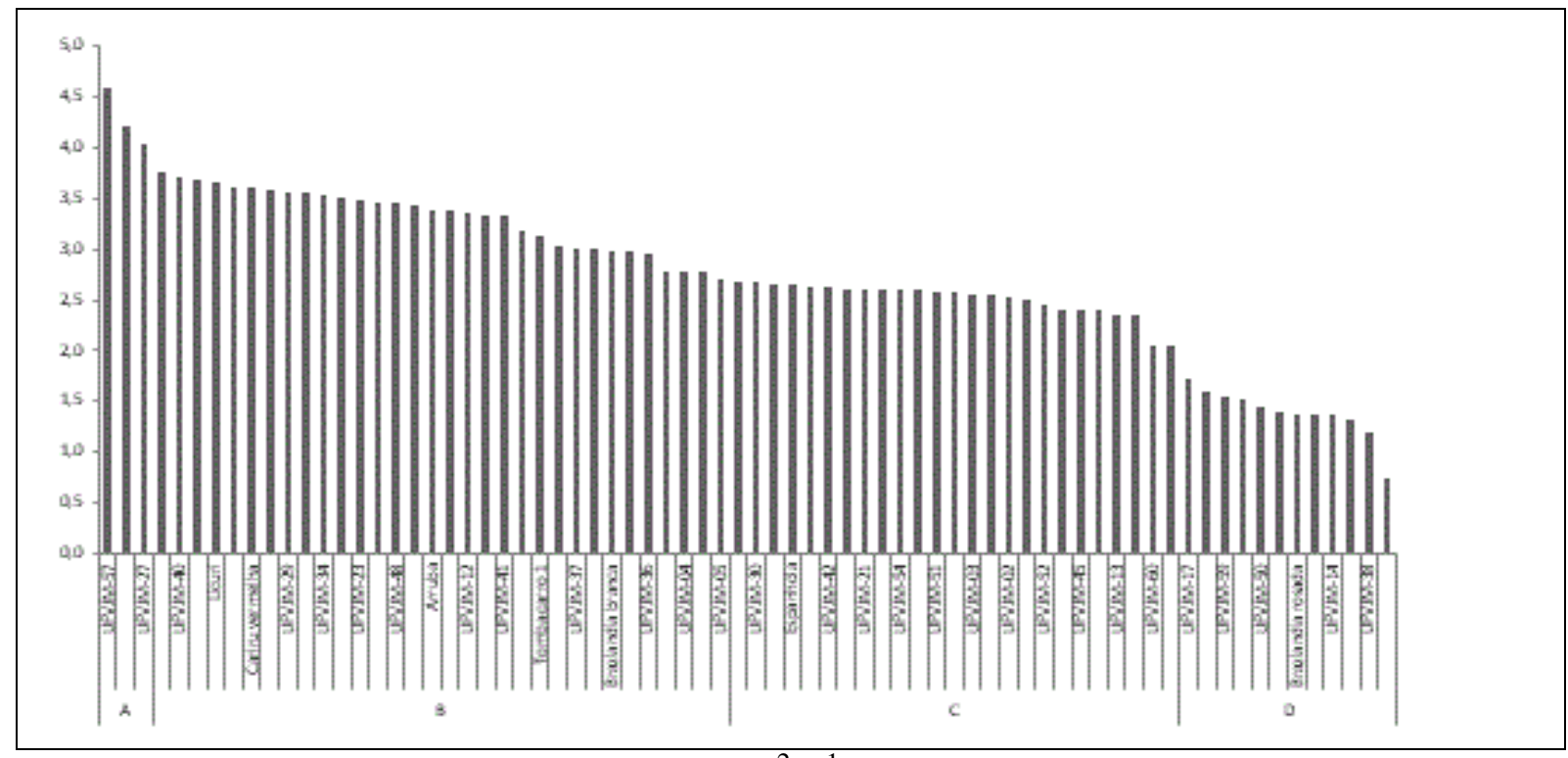

Figura 7. Taxa transpiratória $\left(\mathrm{E}-\mathrm{mol} \mathrm{H}_{2} \mathrm{O} \mathrm{m}^{2} \mathrm{~s}^{-1}\right)$ de setenta e dois genótipos de batata-doce cultivadas no Alto Vale do Jequitinhonha, Diamantina-MG. Médias seguidas pela mesma letra maiúscula em cada barra, não diferem entre si pelo Critério de agrupamento de ScottKnott a 5\% de probabilidade. Transpiratory rate $\left(E-m o l \mathrm{H}_{2} \mathrm{O} \mathrm{m} \mathrm{m}^{2}\right)$ of seventy-seven sweet potato genotypes grown in the Alto Vale do Jequitinhonha, Diamantina-MG. Means followed by the same capital letter in each bar do not differ by Scott-Knott's Grouping Criterion at 5\% probability.

Fonte: Autoria própria. Own authorship. 
Observou-se a formação de quatro grupos referentes à variável taxa transpiratória (E), os valores observados para a variável em questão foram de 4,6 $\mathrm{mol} \mathrm{H}_{2} \mathrm{O} \mathrm{m}{ }^{2} \mathrm{~s}^{-1}$ para UFVJM57 que mostrou o maior valor a $0,8 \mathrm{~mol} \mathrm{H}_{2} \mathrm{O} \mathrm{m}^{2} \mathrm{~s}^{-1}$ para a UFVJM-06, a qual apresentou o valor mais baixo. $\mathrm{O}$ primeiro grupo representado pelos materiais genéticos que mostraram maior E, foram UFVJM-57, UFVJM-01 e UFVJM-27, com valores de E de 4,6; 4,2 e 4 mol $\mathrm{H}_{2} \mathrm{O} \mathrm{m} \mathrm{m}^{2} \mathrm{~s}^{-1}$. No segundo grupo foi constada a presença de 32 materiais genéticos, no grupo 3 observou-se 25 genótipos e no último grupo 12 (Figura 7).

O declínio de transpiração está associado ao fechamento dos estômatos, e variações na abertura estomática causam alterações no potencial hídrico, por atuarem sobre a E (TAIZ et al., 2017). Dessa forma, o funcionamento dos estômatos constitui um comprometimento fisiológico, quando abertos, permitindo a assimilação de gás carbônico. Fechando-se, conservam água e reduzem o risco de desidratação (CONCENÇO et al., 2009), dessa forma, a planta tende a fechar os estômatos quando os níveis de luz estão abaixo da radiação fotossinteticamente ativa, ou para evitar o estresse hídrico. Todos esses parâmetros estão ligados numa relação de custo/benefício, pois a $\mathrm{E}$ também é um mecanismo de diminuição do gradiente entre temperatura da folha e do ar. Os processos de transpiração e captura de $\mathrm{CO}_{2}$ só ocorrem quando os estômatos estão abertos, bem como a Gs. (GALON et al., 2010; GALON et al., 2013; MANABE et al., 2014).

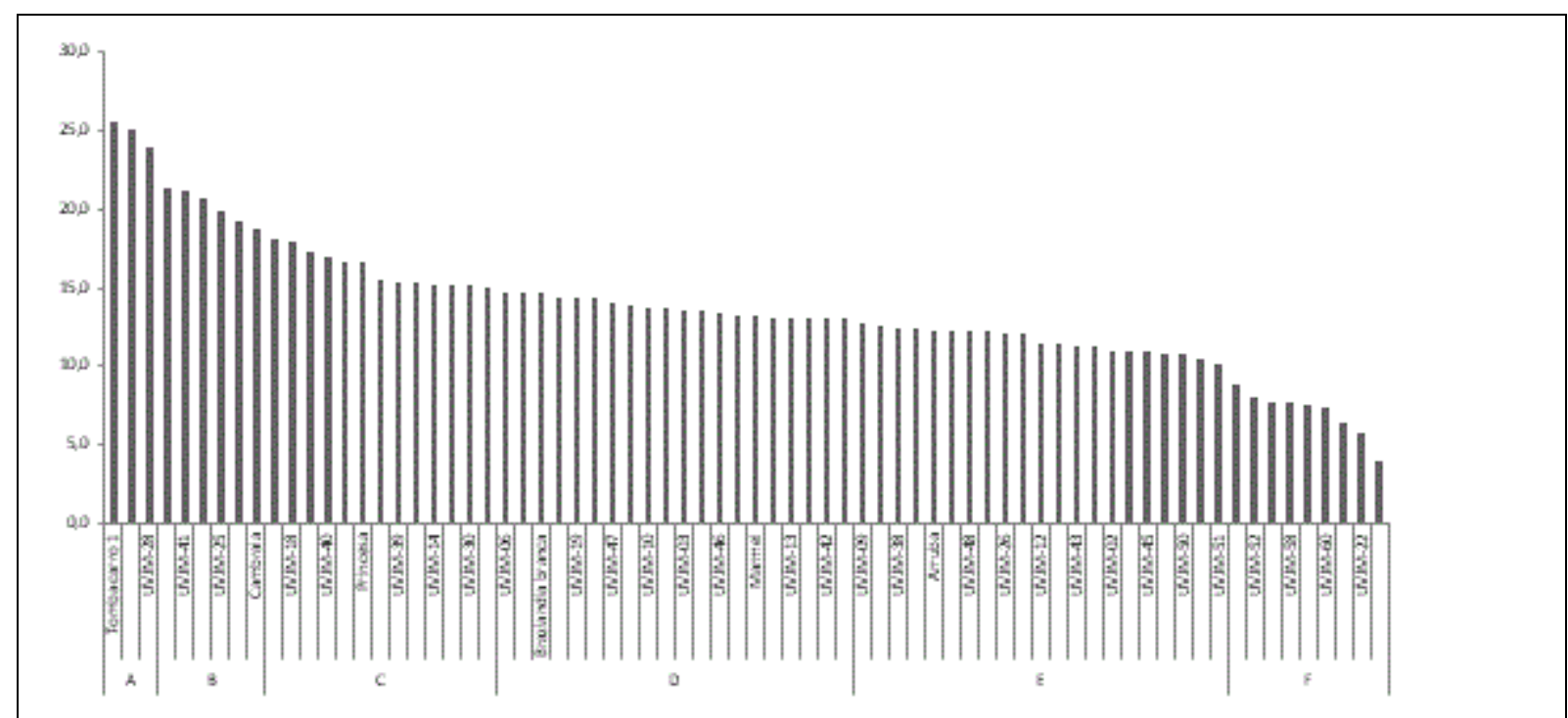

Figura 8. Massa da matéria seca total (MST - g) de setenta e dois genótipos de batata-doce cultivadas no Alto Vale do Jequitinhonha, Diamantina-MG. Médias seguidas pela mesma letra maiúscula em cada barra, não diferem entre si pelo Critério de agrupamento de ScottKnott a 5\% de probabilidade. Mass of total dry matter (MST-g) of seventy-seven sweet potato genotypes grown in the Alto Vale do Jequitinhonha, Diamantina-MG. Means followed by the same capital letter in each bar do not differ by Scott-Knott's Grouping Criterion at 5\% probability.

Fonte: Autoria própria. Own authorship.

Com relação à massa da matéria seca total (MST) contatou-se a formação de seis grupos segundo o critério de agrupamento utilizado. Sendo que, nesses grupos foram observados 3, 6, 13, 20, 21 e 9 genótipos, respectivamente, nos grupos de um a seis. Os genótipos Tomba carro 1 (25,6g), UFVJM-01 (25,0g) e UFVJM-28 (23,9 g) mostraram os maiores valores de MST, 
destacando-se que a cultivar UFVJM-54 mostrou os menores valores referente a esta variável $(3,9 \mathrm{~g})$ (Figura 8).

O material genético UFVJM-57 apresentou os maiores valores de A, Gs, Ci e E, ou seja, com o aumento da taxa fotossintética a demanda por $\mathrm{CO}_{2}$ é maior, desta forma, a condutância estomática também aumenta a fim de capturar $\mathrm{CO}_{2}$, e com o processo intenso de fotossíntese a transpiração também aumentou. Provavelmente devido ao aumento na transpiração o EUA foi menor (grupo C) e consequentemente o genótipo UFVJM-57 obteve valores intermediários de massa.

Já o genótipo UFVJM-01 mostrou valores altos de Gs e E, no caso da E esses valores não diferiram dos observados para a UFVJM-57. Mesmo apresentando taxa fotossintética menor do que a UFVJM-57, a cultiva que o genótipo UFVJM-57, o UFVJM-01 apresentou os maiores valores de massa seca. Uma vez que todos os materiais estiveram sob as mesmas condições hídricas, nutricionais e luminosas supõe-se que as diferenças no acúmulo de matéria seca são provenientes das características intrínsecas de cada genótipo. Por outro lado, o genótipo UFVJM-06 mostrou os menores valores de MST, bem como, os mais baixos valores relacionados às características fisiológicas como A, Gs e E. Resultados semelhantes foram encontrados por Galon et al. (2017) trabalhando com diferentes cultivares de cevada submetidas ao mesmo grau de competição com plantas daninhas, onde concluíram que as cultivares demonstraram capacidade diferente de acúmulo de matéria seca bem como de habilidade competitiva, resultados esses devido as características intrínsecas de cada cultivar.

\section{CONCLUSÃO}

Os genótipos de batata-doce avaliados apresentaram comportamento diferenciado com relação às diversas características fisiológicas estudadas. Destacando-se o acesso UFVJM-57 que apresenta maiores valores de $\mathrm{A}, \mathrm{Gs}, \mathrm{Ci}, \mathrm{Ci} / \mathrm{Ca}$ e E, em contrapartida a UFVJM-06 possui os menores valores com relação a essas características.

\section{AGRADECIMENTOS}

Os autores agradecem ao Conselho Nacional de Desenvolvimento Científico e Tecnológico - CNPq, à Fundação de Amparo à Pesquisa do Estado de Minas Gerais (FAPEMIG) e à Coordenação de Aperfeiçoamento de Pessoal de Nível Superior (CAPES) pelo apoio financeiro na execução deste trabalho.

\section{REFERÊNCIAS BIBLIOGRÁFICAS}

ANDRADE JÚNIOR, V. C.; VIANA, D. J. S.; FERNANDES, J. S. C.; FIGUEIREDO, J. A; NUNES, U. R.; NEIVA, I. P. Selection of sweet potato clones for the region Alto Vale do Jequitinhonha. Horticultura Brasileira, Brasília, v. 27, n. 3, p.389-393, 2009.

ANDRADE JÚNIOR, V. C.; VIANA, D. J. S.; PINTO, N. A. V. D.; RIBEIRO, K. G.; PEREIRA, R. C.; NEIVA, I. P.; AZEVEDO, A. M.; ANDRADE, P. C. R. Características 
produtivas e qualitativas de ramas e raízes de batata-doce. Horticultura Brasileira, Brasília, v. 30, n. 4, p.584-589, 2012.

ANDRADE JÚNIOR, V. C.; PEREIRA, R. C.; DORNAS, M. F. S.; RIBEIRO, K. G.; VALADARES, N. R.; SANTOS, A. A; CASTRO, B. M. C. Produção de silagem, composição bromatológica e capacidade fermentativa de ramas de batata-doce emurchecidas. Horticultura Brasileira, Brasília, v. 32, n. 1, p.91-97, 2014.

CONCENÇO, G.; FERREIRA, E. A.; SILVA; A. A.; FERREIRA, F. A.; VIANA, R. G.; D'ANTONINO, L.; VARGAS, L.; FIALHO, C. M. T. Uso da água em biótipos de azevém (Lolium multiflorum) em condição de competição. Planta Daninha, Viçosa, v. 25, n. 3, p.449-455, 2007.

CONCENÇO, G.; FERREIRA, E. A.; SILVA, A. A.; FERREIRA, F. A.; GALON, L.; REIS, M. R.; d'ANTONINO, L.; VARGAS, L.; SILVA, L. V. B. D. Fotossíntese de biótipos de azevém sob condição de competição. Planta Daninha, Viçosa, v. 26, n. 3, p.595-600, 2008.

CONCENÇO, G.; FERREIRA, E. A.; ASPIAZU, I.; SILVA, A. F.; GALON, L.; FERREIRA, F. A.; SILVA, A. A.; ANDRES, A. Uso da água por plantas de arroz em competição com biótipos de Echinochloa crusgalli resistente e suscetível ao herbicida quinclorac. Planta Daninha, Viçosa, v. 27, n. 2, p.249-256, 2009.

FERREIRA, E. A.; ASPIAZÚ, I.; CONCENÇO, G.; SILVA, A. F.; SILVA. A. A.; LEANDRO L GALON, L. L.; SILVA, D. V. Evaluation and grouping of sugarcane genotypes in agreement with their physiologic characteristics types. Revista Trópica: Ciências Agrárias e Biológicas, Chapadinha, v. 5, n. 3, p.30-38, 2011.

GALON, L.; FERREIRA, E. A.; CONCENÇO, G.; SILVA, A. A.; SILVA, D. V.; SILVA, A. F.; ASPIAZÚ, I.; VARGAS, L. Características fisiológicas de biótipos de Conyza bonariensis resistentes ao glyphosate cultiva dos sob competição. Planta Daninha, Viçosa, v. 31, n. 4, p.859-866, 2013.

GALON, L.; CONCENÇO, G.; FERREIRA, E. A.; ASPIAZÚ, I.; SILVA, A. F.; FERREIRA, F. A.; SILVA, A. A.; TIRONI, S. P.; FREITAS, M. A. M.; SOARES, E. R. Eficiência de uso da água em genótipos de cana-de-açúcar submetidos à aplicação de herbicidas. Planta Daninha, Viçosa, v. 28, n. 4, p.777-784, 2010.

GALON, L.; AGAZZI, L. R.; NONEMACHER, F.; BASSO, F. J. M.; WINTER, F. L.; FIABANE, R. C.; ZABOT, G. F.; PERIN, G. F. Competitive relative ability of barley cultivars in interaction with turnip. Planta Daninha, Viçosa, v. 35, n. e017164016, p.1-10, 2017.

MANABE, P. M. S.; MATOS, C. C.; FERREIRA, E. A.; SILVA, A. A.; SEDIYAMA, T.; MANABE, A.; SILVA, A. F.; ROCHA, P. R. R.; GALON, L. Características fisiológicas de feijoeiro em competição com plantas daninhas. Bioscience Journal, Uberlândia, v. 30, n. 6, p. 1721-1728, 2014.

PINZÓN-TORRES, J. A.; SCHIAVINATO, M. A. Crescimento, eficiência fotossintética e eficiência do uso da água em quatro espécies de leguminosas arbóreas tropicais. Hoehnea, São Paulo, v. 35, n. 3, p.395-404, 2008. 
RUKUNDO, P.; HUSSEIN, S.; MARK, L.; DAPHROSE, G. Storage root formation, dry matter synthesis, accumulation and genetics in sweetpotato. Australian Journal of Crop Science, Lismore, v. 7, n. 13, p.2054-2061, 2013.

SANTANA, W.R.; MARTINS, L. P.; SILVEIRA, M. A.; SANTOS, W. F.; GONÇALVES, R. C.; SOUZA. F. R.; RESPLANDES, G. R. S.; LIMA, M. D. L. Identificação agronômica de genótipos de batata-doce em banco de germoplasma para fins industriais de etanol carburante. Revista Tecnologia \& Ciência Agropecuária, João Pessoa, v. 7, n. 1, p.31-34, 2013.

SANTOS, M.C.; NUSSIO, L. G.; MOURÃO G. B.; SCHMIDT, B.; MARI, L. J.; RIBEIRO, J. L.; QUEIROZ, O. C. M.; ZOPOLLATTO, M.; SOUSA, D. P.; SARTURI, J. O.; TOLEDO FILHO, S. G. Nutritive value of sugarcane silage treated with chemical additives. Scientia Agricola, Piracicaba, v. 66, n. 2, p.159-163, 2009.

SILVA, A. A.; FERREIRA, F. A.; FERREIRA, L. R.; SANTOS, J. B. Biologia de plantas daninhas. In: SILVA, A. A.; SILVA, J. F. (ed.) Tópicos em manejo de plantas daninhas. Viçosa, MG: Editora UFV, 2012. cap.1, p. 1-40.

TAIZ, L.; MØLLER, I. M.; MURPHY, A. S.; ZEIGER, E. Fisiologia e desenvolvimento vegetal. 6. ed. Porto Alegre: Artmed, 2017. 888 p.

VARGAS, P. F.; GODOY, D. R. Z.; ALMEIDA, L. C. F.; RENATA CASTOLDI, R. Agronomic characterization of sweet potato accessions. Comunicata Scientiae, Bom Jesus, v. 8, n. 1, p.116-125, 2017. 